\title{
Evaluación Integral en docentes. Perfiles de docentes con respecto su percepción de la evaluación
}

\author{
Comprehensive Assessment of Teachers. Profiles of Teachers Regarding their Perception \\ of the Assessment
Avaliação Integral em professores. Perfis dos professores em torno da percepção da avaliação

\author{
Elvira Carpintero M., ${ }^{a}$ Coral González B. ${ }^{b}$ y Diana Cabezas . $^{c}$
}

${ }^{\text {a}}$ Dpto. Métodos de Investigación y Diagnóstico en Educación, Universidad Complutense de Madrid, España. Telf.: 91 3946147. Correo electrónico: ecarpintero@edu.ucm.es

bDpto. Métodos de Investigación y Diagnóstico en Educación, Universidad Complutense de Madrid, España. Telf.: 91394 6149. Correo electrónico: cgbarbera@edu.ucm.es

cAsociación Gorabide, Bizkaia, España. Tfno. 94 4431210. Correo electrónico:dianacabezas@gorabide.com

\section{RESUMEN}

Las creencias sobre cualquier hecho educativo se relacionan, sin duda, con la práctica del mismo. En concreto, las ideas que los docentes tienen sobre la evaluación, su función y modalidades, se vinculan con el modo de hacer en el aula. El propósito del presente trabajo es analizar las prácticas de los docentes con respecto a la evaluación comparando sus diferencias en función de algunas variables sociodemográficas. Para ello, se dispuso de una muestra de 175 docentes que completaron el Cuestionario de Evaluación Integral, compuesto por 59 ítems que corresponden a seis dimensiones del mismo y que se refieren a las distintas formas de entender y practicar la evaluación: integradora, contextualizada, formativa, reflexiva, democrática y personalizada. Los resultados mostraron diferencias en las formas de concebir la evaluación por parte de los docentes en función de variables como el sexo, edad, de la etapa educativa y la formación académica de los mismos.

Palabras clave: evaluación, evaluación integral, educación primaria, educación secundaria, teorías implícitas.

\begin{abstract}
The beliefs about any educative fact are related to its practice. Specifically, the ideas that teachers have about function and modality of assessment are linked with the way they do in the classroom. The purpose of this paper is to analyze the practices of teachers regarding to assessment, comparing their differences based on demographic variables. The final sample selected through incidental sampling, consisted of 175 teachers who completed the Comprehensive Assessment Questionnaire, which consisted of 59 items that corresponded to six dimensions of it, that means, that were related to different ways of understanding and practicing assessment: integrative, contextualized, training, reflexive, democratic and personalized. The results showed differences in the way teachers conceive assessment in terms of variables such as sex, age, educational stage and academic training on them.

Key words: assessment, comprehensive educational assessment, elementary education, secondary education, implicit theories.

\section{RESUMO}

Crenças sobre qualquer ação educativa se relacionam, sem dúvida, com a prática da mesma. Especificamente, as idéias que os professores têm sobre a avaliação, suas funções e moda-lidade, vinculam-se com a prática da sala de aula. Objetiva-se analisar as práticas docentes em relação à avaliação, comparando-se as diferenças relaciondas às variáveis sociodemográficas. Para tanto, partiu-se de uma amostra de 175 professores que completaram o Questionário
\end{abstract}


de Avaliação Integral, composto por 59 itens que correspondem a seis dimensões do mesmo e que se referem à diferentes formas de compreensão e prática da avaliação: integradora, con-textualizada, formativa, reflexiva, democrática e personalizada. Resultados mostraram, por parte dos professores, diferenças nas maneiras de conceber a avaliação em relação às variáveis como: sexo, idade, níveis educativos e formação acadêmica dos mesmos.

Palavras chave: avaliação, avaliação integral, educação primária, educação secundária, teorias implícitas.

\section{INTRODUCCIÓN}

Uno de los elementos que mayor controversia genera en el proceso educativo es la evaluación. Se trata de un elemento fundamental y complejo que condiciona el propio proceso de la educación (Rosales, 2000; Salinas, 2002; Santos, 2003) y que, por tanto, requiere de una profunda reflexión tanto en torno a parámetros de carácter teórico como prácticos y éticos.

Para De la Orden (1989), la evaluación condiciona lo que los alumnos aprenden y cómo lo aprenden, y lo que los profesores enseñan y cómo lo enseñan, de modo que la actividad educativa de alumnos y profesores se encuentra mediada por la evaluación. De hecho, a través de la evaluación se puede conocer la manera en que los docentes entienden la educación y el concepto que tienen de la misma (Coll, Martín y Onrubia, 2001).

La complejidad del proceso estriba, entre otros motivos, en la variedad de los distintos elementos que la articulan. Además de los elementos explícitos (especificaciones administrativas y legales, consideraciones de los propios centros escolares, condicionamientos de las aulas y los grupos de alumnos) existen un conjunto de elementos implícitos que participan en su configuración. Así, las concepciones que los docentes tienen sobre la enseñanza en general y la evaluación en particular, la importancia y consecuencias de la misma, los instrumentos elegidos o elegibles para llevarla a cabo, las consideraciones sobre su finalidad y su objetivo, además de la propia experiencia, son esenciales a la hora de entender los procesos de evaluación que llevan a cabo los docentes en sus aulas.

Las acciones y prácticas de evaluación tienen importantes repercusiones, no solo sobre el rendimiento y posterior promoción del alumno, sino también sobre otros aspectos de su desarrollo, tales como su personalidad y su autoestima. Entre otros elementos, la imagen que los alumnos construyen de sí mismos como mejores o peores estudiantes puede llegar a estar influida por la experiencia personal de evaluación que han vivido lo que, sin duda, incidirá en su futuro profesional (Litwin, 2005). Además, a través de los procesos de evaluación, los estudiantes desarrollan determinados valores y constituyen ciertas ideas y juicios, pues participan en situaciones en las que se evidencian determinadas formas de afrontar la realidad y responder ante ésta. Así, según Pérez y González (2011), a través de los procesos de evaluación es posible desarrollar una determinada concepción de la justicia social, tomando como punto de partida la clasificación de Perrenoud (2008) que distingue entre una evaluación centrada en la diferenciación y en la fabricación de jerarquías y una evaluación centrada en la regulación de los aprendizajes.

El primer tipo de evaluación se caracteriza por la presencia pasiva del alumno que es evaluado únicamente por el docente a través de pruebas y herramientas estandarizadas que permite la clasificación y jerarquización de los sujetos; se produce, por tanto, un énfasis en los resultados en detrimento de los procesos. Por otro lado, la evaluación centrada en la regulación de los aprendizajes presenta al alumno como sujeto de evaluación y ésta se presenta como una tarea colaborativa con el docente, de tal forma que se centra 
esencialmente en los procesos de forma continua. Los autores establecen una posible relación entre el primer tipo de evaluación y un concepto de justicia social caracterizado por la imparcialidad y la igualdad de oportunidades, con el mérito como principal criterio para el establecimiento de diferencias, frente a un concepto de justicia social vinculado al reconocimiento de las diferencias y desigualdades, y donde no existe un juicio único y objetivo, sino que éste se construye a partir de un diálogo, elaborado desde prácticas de evaluación centradas en la regulación del aprendizaje.

Desde la actual legislación educativa en España, la evaluación se entiende como un instrumento valioso para el seguimiento y valoración de los resultados, así como para la mejora de los procesos. En la etapa de Educación Primaria, la evaluación se realizará de forma continua y global, atendiendo al progreso del alumno en el conjunto de las áreas de aprendizaje. En Educación Secundaria Obligatoria y Bachillerato, la evaluación será igualmente continua y diferenciada según las distintas materias del currículo. Por otro lado, se incorpora una evaluación de diagnóstico de las competencias básicas de los alumnos al finalizar el segundo ciclo de Primaria, y el segundo curso de la ESO, con carácter formativo y orientador (LOE, 2006).

Sin embargo, y a pesar de que la legislación educativa ampara y promueve formas amplias y adaptadas de evaluar, y que entre las funciones del profesorado se incluye la evaluación de los procesos de aprendizaje de los alumnos, no siempre ésta se interpreta como un proceso de mejora (o como un elemento de ayuda y mejora), y por ello, conviene reflexionar sobre tal y como indica Sanmartí (2007): “dime qué y cómo evalúas y te diré qué y cómo enseñas y qué y cómo tus alumnos aprenden” (19).

Tomando como elemento de partida esta reflexión de Sanmartí, parece importante analizar cuáles son los procesos de evaluación que llevan a cabo los docentes y qué consideraciones tienen al respecto. El análisis de los procesos de evaluación nos llevará, sin duda, a conocer con mayor detalle los procesos sobre la educación. Así, la forma de plantear y llevar a cabo la evaluación evidencia, no solo particularidades de carácter técnico y pedagógico, sino también, según algunos autores, significaciones de tipo político, social y moral en el profesor (Santos, 2003).

Por ello, los planteamientos y acciones de evaluación manifiestan consideraciones sobre otros elementos relativos al proceso educativo (concepción de la educación, modelos de promoción de los alumnos, aspectos relacionados con las formas de aprendizaje, etc.).

Las creencias sobre la evaluación se relacionan con la práctica sobre la misma. Así, aquellos docentes que entienden la evaluación como un proceso de control, buscan la consecución de respuestas correctas en los alumnos; por el contrario, para aquellos que entienden la evaluación como un proceso para comprender y mejorar su propia docencia, buscan la comprensión más que la reproducción y la participación de los alumnos (Prieto y Contreras, 2008).

Sin embargo, los estudios sobre la motivación, actitudes y pensamientos que acompañan a la evaluación que realizan los docentes no son muy numerosos (Blanco y González, 2010). Cabe señalar, no obstante, el estudio llevado a cabo por Brown (2004) quien, a través de un cuestionario, evaluó a más de 500 profesores de Educación Primaria de Nueva Zelanda sobre sus concepciones de la evaluación, hallando que dichos docentes consideraron la evaluación como un elemento de importancia tanto para la enseñanza como para el aprendizaje de los alumnos, así como para el conocimiento del funciona- 
miento escolar. Sin embargo, no se hallaron diferencias estadísticamente significativas en función del sexo, formación académica o experiencia docente entre los participantes.

Por otro lado, Vergara (2011) realiza un interesante estudio centrado en las concepciones sobre la evaluación del aprendizaje de ciertos docentes que son evaluados con un desempeño óptimo en su práctica diaria a través de un estudio de casos, con objeto de describir y comprender cuáles fueron sus prácticas de evaluación. Los cuatro docentes participantes estuvieron de acuerdo en considerar la evaluación como una parte esencial del proceso de enseñanza-aprendizaje, destacando la relación entre las actividades de aprendizaje y las actividades de evaluación; además, reconocieron la importancia que la participación del alumno tiene a lo largo del proceso, tanto con la auto como con la coevaluación. Por último, los docentes describieron la evaluación, no como un obstáculo de su práctica educativa, sino como una tarea que posibilita la mejora de sus propias estrategias metodológicas.

Entre los estudios con población española, Martín et al. (2006), en una investigación llevada a cabo con docentes de Educación Primaria, observaron que los docentes con menos años de experiencia seleccionan consideraciones de la evaluación donde primaba el papel activo y constructivo de la misma, frente a los docentes de mayor edad quienes consideraban la evaluación como un instrumento para registrar el conocimiento y donde el papel del alumno era más pasivo y principalmente reproductivo. En esta misma línea se encuentran los resultados de Bautista, Pérez y Pozo (2011) en un estudio con profesores de piano quienes hallaron que los docentes con menos años de experiencia (menos de 5 años) consideraban en sus evaluaciones elementos dirigidos a valorar tanto los resultados como los procesos de aprendizaje de los alumnos, a diferencia de los docentes con más años de experiencia (más de 15 años) quienes principalmente comprobaban los resultados de los alumnos, sin promover procesos de reflexión y autovaloración.

El término "evaluación integral" es una expresión actualmente utilizada y vinculada al mundo empresarial y se halla relacionada con una metodología de análisis de situaciones y hechos con objeto de encontrar soluciones eficaces. En este sentido, trata de evaluar los distintos procesos que configuran una empresa con objeto de favorecer la eficiencia, la productividad y la competitividad de la misma (Fleitman, 2007). Según García, Barría, Prieto, Herrera y Vázquez (2006), la evaluación integral es una metodología por medio de la cual se estudian, analizan y evalúan las fuerzas, debilidades, amenazas y oportunidades de las empresas. En este sentido, uno de los grandes valores de la evaluación integral es que permite observar las situaciones desde la perspectiva de oportunidades futuras; por lo tanto, la importancia de esta evaluación radica en la posibilidad de modificar los procesos inadecuados y mejorarlos (Fleitman, 2007).

Desde el ámbito educativo, la evaluación integral debe dirigirse a responder a las necesidades, tanto de los propios estudiantes como del profesorado y de los centros. Así, intenta dar respuesta, al menos, a los siguientes elementos: la evaluación de los profesores, la evaluación de los centros y la evaluación del sistema educativo.

Tal y como recoge Pérez Juste (2002), si buscamos y defendemos una educación integral, no puede optarse por una posición reduccionista en la evaluación, sino que ésta debe ser, asimismo, evaluación integral.

En esta línea, y con objeto de implicar a los docentes en un proceso de reflexión sistemática sobre la evaluación, se plantea la presente investigación, partiendo de las ideas previas que dichos docentes tienen sobre la evaluación, esto es, realizando un análisis de 
sus teorías implícitas (Carpintero y Cabezas, 2007; Cabezas y Carpintero 2006). Como consecuencia de dicho análisis, se elabora un Cuestionario de Evaluación Integral, que con dicha nominación pretende recoger la variedad de prácticas de evaluación que se realizan en el aula. Tras la validación del instrumento se procedió al estudio de las distintas respuestas de un grupo de docentes de distintos centros educativos.

\section{MÉTODO}

\subsection{OBJETIVO GENERAL}

Analizar las prácticas de los docentes con respecto a la evaluación comparando sus diferencias en función de algunas variables sociodemográficas.

\subsection{MUESTRA}

La muestra final, elegida mediante muestreo incidental, estuvo compuesta por 175 docentes, pertenecientes a cuatro centros públicos de distintas zonas del territorio español. ${ }^{1}$

En función del género, más del $75 \%$ eran mujeres, siendo únicamente algo más del $20 \%$ varones.

Respecto a la edad de los participantes, la mayoría contaban entre 30 y 40 años (43.4\%), siendo el porcentaje inferior (10.3\%) el de los docentes con más de 50 años. En el extremo inferior de edad se encontraban los docentes menores de 30 años (24\%), tal y como se muestra en la Figura 1.

En cuanto al nivel de estudios de los docentes, éstos presentaban niveles de diplomatura y licenciatura en porcentajes equivalentes. Las distintas etapas en las que impartían su docencia se muestran en la Tabla 1.

Respecto a la experiencia docente, menos del $20 \%$ contaban con experiencias inferiores a 4 años, por lo que la gran mayoría presentaba una experiencia superior a dicha cifra (Figura 2).

Figura 1. Distribución de la muestra según la edad

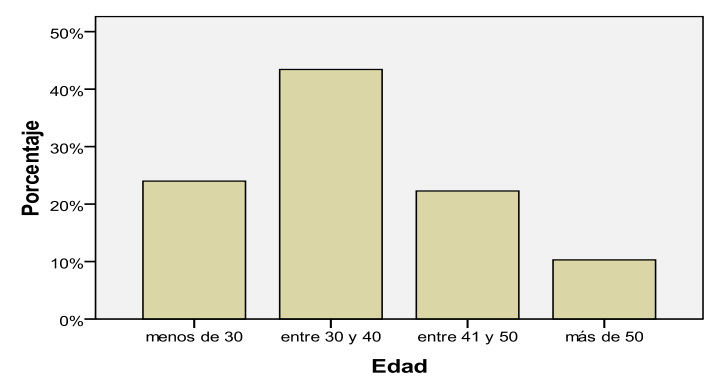

1 En concreto, participaron los siguientes centros educativos: Colegio Juan de Lanuza (Zaragoza), Colegio Ramón y Cajal (Madrid), Grupo Sorolla (Valencia) y Centro Educativo y Deportivo LLedó (Castellón). 
Tabla 1. Distribución de la muestra en función de la etapa educativa en la que imparten su docencia

\begin{tabular}{|c|c|c|}
\hline & Frecuencia & Porcentaje \\
\hline Primer ciclo EI & 21 & 12,0 \\
\hline Segundo ciclo EI & 36 & 20,6 \\
\hline Educación Primaria & 50 & 28,6 \\
\hline Educación Secundaria Obligatoria & 47 & 26,9 \\
\hline Bachillerato & 21 & 12,0 \\
\hline
\end{tabular}

Figura 2. Distribución de la muestra según su experiencia docente

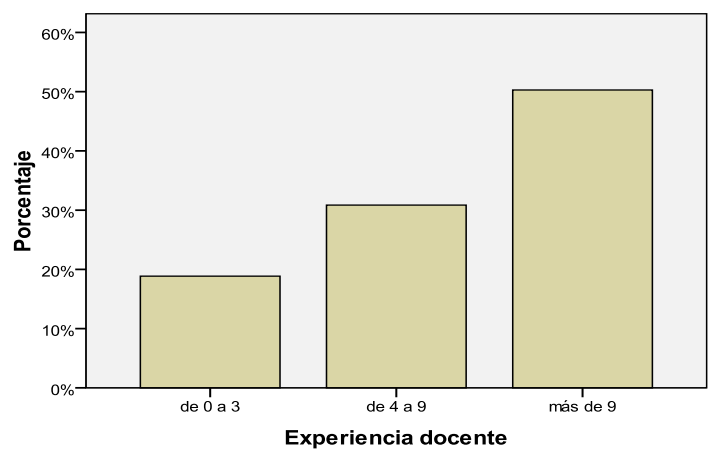

\subsection{INSTRUMENTO}

El Cuestionario de Evaluación Integral (CEI) surge como demanda de un grupo de profesionales del ámbito de la educación ${ }^{2}$ comprometidos y preocupados por el alcance de la evaluación y las repercusiones de la misma. Dicho grupo se propuso reflexionar sobre qué implica la evaluación y cuáles son las áreas fundamentales que deben ser valoradas, seleccionando el término evaluación integral.

Para ello, se procedió a elaborar y validar dicho instrumento, obteniendo una fiabilidad de 0.868 , calculada a través del índice de consistencia interna alfa de Cronbach (más detalles sobre la validación del CEI pueden encontrarse en Cabezas, González y Carpintero, 2009).

El cuestionario CEI está compuesto por 59 ítems que corresponden a las seis dimensiones del mismo (ver tabla 2). La forma de respuesta del fue mediante escala Likert graduada de 1 a 4 .

2 El equipo de trabajo estuvo conformado por Cendegui, M., Del Lozar, E., Díaz, A., García, G., Laliga, A., López, P., Moreno, L. y Redondo, S. 
Tabla 2. Dimensiones teóricas del Cuestionario CEI

\begin{tabular}{|c|c|}
\hline DIMENSIONES & DESCRIPCIÓN \\
\hline $\begin{array}{l}\text { I: Evaluación } \\
\text { integradora }\end{array}$ & $\begin{array}{l}\text { Hace referencia a los diversos aspectos propios del alumno a la hora de evaluar su } \\
\text { proceso de aprendizaje: aptitudes, actitudes, intereses, aspectos de personalidad, etc. }\end{array}$ \\
\hline $\begin{array}{l}\text { II: Evaluación } \\
\text { contextualizada }\end{array}$ & $\begin{array}{l}\text { Hace referencia a los diversos aspectos que pueden afectar el proceso de evaluación } \\
\text { y que deben, a su vez, estar en consonancia con la misma: práctica docente, } \\
\text { currículo, centro, aula, familia, etc. }\end{array}$ \\
\hline $\begin{array}{l}\text { III: Evaluación } \\
\text { formativa }\end{array}$ & $\begin{array}{l}\text { Hace referencia a los momentos de la evaluación: inicial, procesual o continua y } \\
\text { final. }\end{array}$ \\
\hline $\begin{array}{l}\text { IV: Evaluación } \\
\text { reflexiva }\end{array}$ & $\begin{array}{l}\text { Hace referencia a los objetivos o propósitos de la evaluación: regulación de los } \\
\text { procesos de EA, informar al propio alumno y su familia, calificar, promocionar, etc. }\end{array}$ \\
\hline $\begin{array}{l}\text { V: Evaluación } \\
\text { democrática }\end{array}$ & $\begin{array}{c}\text { Hace referencia a los procedimientos de evaluación: heteroevaluación, } \\
\text { autoevaluación y coevaluación }\end{array}$ \\
\hline $\begin{array}{l}\text { VI: Evaluación } \\
\text { personalizada }\end{array}$ & $\begin{array}{l}\text { Hace referencia a las diversas técnicas e instrumentos de los que se ayuda el docente } \\
\text { en su práctica evaluativa }\end{array}$ \\
\hline
\end{tabular}

\subsection{PROCEDIMIENTO}

La aplicación se llevó a cabo en cada uno de los centros educativos indicados, coordinada por los respectivos Departamentos de orientación. Los cuestionarios se distribuyeron entre los docentes de Infantil, Primaria, Secundaria y Bachillerato y se completaron de manera anónima, con unas indicaciones comunes en todos los centros, consensuadas previamente por el grupo de trabajo.

\subsection{ANÁLISIS DE DATOS}

Para la realización de los análisis estadísticos se empleó el paquete estadístico Statistical Package for Social Sciences (SPSS) versión 19 para Windows. Se realizó una prueba t para muestras independientes con objeto de valorar las posibles diferencias en la concepción de la evaluación integral y sus distintas dimensiones en función del sexo. Para estudiar las diferencias en función de la edad, de la etapa educativa y la formación académica de los docentes se realizaron distintos Análisis de Varianza.

\section{RESULTADOS}

El planteamiento inicial de la investigación no esperaba hallar diferencias en la concepción de la evaluación en función del sexo de los profesionales de la educación, sin embargo, se hallaron diferencias en cuatro de las seis dimensiones de la prueba CEI: evaluación integradora $(\mathrm{t}=-3.199, \mathrm{p}=0.002)$, evaluación contextualizada $(\mathrm{t}=-3.080, \mathrm{p}=$ $0.002)$, evaluación formativa $(\mathrm{t}=2.985, \mathrm{p}=0.003)$ y evaluación personalizada $(\mathrm{t}=-3.489$, $\mathrm{p}=0.001$ ). Así, en la evaluación formativa, los docentes puntuaron significativamente más alto que las mujeres, mientras que en las dimensiones integradora, contextualizada y personalizada fueron las mujeres las que presentaron puntuaciones superiores. Por otro lado, en las dimensiones de evaluación reflexiva y evaluación democrática no se hallaron diferencias estadísticamente significativas (Tabla 3). 
Tabla 3. Resultados en función del sexo

\begin{tabular}{|c|c|}
\hline DIMENSIONES & $\begin{array}{c}\text { DIFERENCIAS ESTADÍTICAMENTE } \\
\text { SIGNIFICATIVAS }\end{array}$ \\
\hline Evaluación integradora & $\begin{array}{c}\text { Diferencias a favor de las mujeres. } \\
(\mathrm{t}=-3.199, \mathrm{p}=0.002)\end{array}$ \\
\hline Evaluación contextualizada & $\begin{array}{c}\text { Diferencias a favor de las mujeres. } \\
(\mathrm{t}=-3.080, \mathrm{p}=0.002)\end{array}$ \\
\hline Evaluación formativa & $\begin{array}{c}\text { Diferencias a favor de los hombres. } \\
(\mathrm{t}=2.985, \mathrm{p}=0.003)\end{array}$ \\
\hline Evaluación reflexiva & No se hallaron diferencias significativas \\
\hline Evaluación democrática & No se hallaron diferencias significativas \\
\hline Evaluación personalizada & $\begin{array}{c}\text { Diferencias a favor de las mujeres. } \\
(\mathrm{t}=-3.489, \mathrm{p}=0.001)\end{array}$ \\
\hline
\end{tabular}

Por otro lado, y de forma coherente con investigaciones anteriores, se esperaba que los docentes de mayor edad presentaran concepciones diferenciadas frente a los docentes más jóvenes, concretamente se esperaban puntuaciones superiores en la dimensión referida a la evaluación formativa, esto es, con los momentos en los que debe procederse a la evaluación, y en la evaluación reflexiva, es decir, el uso de la evaluación como instrumento para compartir información sobre la valoración y progreso del alumno. Sin embargo, los resultados obtenidos tampoco confirmaron esta hipótesis ya que no se obtuvieron diferencias estadísticamente significativas en dichas dimensiones. Por el contrario, se hallaron diferencias estadísticamente significativas entre las dimensiones de la evaluación integradora $(F=3.600, p=0.015)$, contextualizada $(\mathrm{F}=6.284, \mathrm{p}=0.000)$ y personalizada $(\mathrm{F}=3.554, \mathrm{p}=0.016)$. Estas diferencias fueron especialmente significativas entre los docentes menores de 30 años y los docentes entre 30 y 40, y entre 41 y 50 años, respectivamente, tanto en la dimensión de evaluación integradora como en la dimensión de evaluación contextualizada. Para la dimensión de la evaluación personalizada se hallaron diferencias significativas entre los docentes menores de 30 años y los docentes cuyo rango de edad comprendía entre 41 y 50 años. En todos los casos, las puntuaciones superiores correspondieron a los docentes menores de 30 años (ver Tabla 4).

Tabla 4. Perfiles en función de la edad

\begin{tabular}{|c|c|}
\hline DIMENSIONES & $\begin{array}{c}\text { DIFERENCIAS ESTADÍTICAMENTE } \\
\text { SIGNIFICATIVAS }\end{array}$ \\
\hline Evaluación integradora & $\begin{array}{c}\text { Entre menores de } 30 \text { años y los entre } 30 \mathrm{y} 40, \mathrm{y} \\
\text { entre } 41 \mathrm{y} 50 \text { años }(\mathrm{F}=3.600, \mathrm{p}=0.015)\end{array}$ \\
\hline Evaluación contextualizada & $\begin{array}{c}\text { Entre menores de } 30 \text { años y los entre } 30 \mathrm{y} 40, \mathrm{y} \\
\text { entre } 41 \mathrm{y} 50 \text { años }(\mathrm{F}=6.284, \mathrm{p}=0.000)\end{array}$ \\
\hline Evaluación formativa & No se hallaron diferencias significativas \\
\hline Evaluación reflexiva & No se hallaron diferencias significativas \\
\hline Evaluación democrática & No se hallaron diferencias significativas \\
\hline Evaluación personalizada & Entre menores de $30 \mathrm{y}$ entre 41 y 50 años. $(\mathrm{F}=$ \\
$3.554, \mathrm{p}=0.016)$
\end{tabular}


Por último, cabe señalar con respecto a los docentes cuya edad superaba los 50 años, que no se hallaron diferencias estadísticamente significativas en ninguna de las dimensiones de la prueba, probablemente debido al escaso tamaño de dicha muestra.

Otra de las hipótesis de estudio se centraba en el análisis de las distintas prácticas y concepciones de evaluación en función del grado de formación académica de los docentes participantes. En este sentido, se consideró que los docentes con formación media, esto es, diplomados en Magisterio, manifestarían puntuaciones superiores en las dimensiones integradora y contextualizada, mientras que los docentes con formación superior, es decir, licenciados, puntuarían más alto en las dimensiones democrática y formativa, hipotetizando además que no habría diferencias en las dimensiones reflexiva y personalizadora. Los resultados mostraron que dichas diferencias fueron estadísticamente significativas para las dimensiones integradora $(\mathrm{F}=5.480, \mathrm{p}=0.001)$, contextualizada $(\mathrm{F}=$ 9.641, $\mathrm{p}=0.000)$, formativa $(\mathrm{F}=13.512, \mathrm{p}=0.000)$ y personalizada $(\mathrm{F}=3.152, \mathrm{p}=0.026)$, cumpliendo así parcialmente la hipótesis. Para las dimensiones integradora, contextualizada y personalizada las puntuaciones fueron superiores para los docentes diplomados, mientras que para la dimensión formativa la puntuación superior correspondió con los docentes licenciados (ver Tabla 5).

Tabla 5. Perfiles en función de la formación académica

\begin{tabular}{|c|c|}
\hline DIMENSIONES & $\begin{array}{c}\text { DIFERENCIAS ESTADÍTICAMENTE } \\
\text { SIGNIFICATIVAS }\end{array}$ \\
\hline Evaluación integradora & $\begin{array}{c}\text { Diferencias a favor de los diplomados } \\
(\mathrm{F}=5.480, \mathrm{p}=0.001)\end{array}$ \\
\hline Evaluación contextualizada & $\begin{array}{c}\text { Diferencias a favor de los diplomados } \\
(\mathrm{F}=9.641, \mathrm{p}=0.000)\end{array}$ \\
\hline Evaluación formativa & $\begin{array}{c}\text { Diferencias a favor de los licenciados } \\
(\mathrm{F}=13.512, \mathrm{p}=0.000)\end{array}$ \\
\hline Evaluación reflexiva & No se hallaron diferencias significativas \\
\hline Evaluación democrática & No se hallaron diferencias significativas \\
\hline Evaluación personalizada & $\begin{array}{c}\text { Diferencias a favor de los diplomados } \\
(\mathrm{F}=3.152, \mathrm{p}=0.026)\end{array}$ \\
\hline
\end{tabular}

Dado que el grado de formación académica de los docentes se halla, frecuentemente, vinculado con la etapa educativa en la que imparten su docencia, la hipótesis de trabajo inicial se describió en términos semejantes a la hipótesis relacionada con la formación académica. Los resultados hallados fueron consistentes con los datos anteriores pues se obtuvieron, nuevamente, diferencias estadísticamente significativas en las dimensiones de evaluación integradora $(\mathrm{F}=9.969, \mathrm{p}=0.000)$, evaluación contextualizada $(\mathrm{F}=11.527$, $\mathrm{p}=0.000)$, evaluación formativa $(\mathrm{F}=22.339, \mathrm{p}=0.000)$ y evaluación personalizada $(\mathrm{F}=$ $5.914, \mathrm{p}=0.000)$.

A través de la prueba post hoc de Tukey se halló que estas diferencias fueron estadísticamente significativas entre los docentes de Educación Infantil y los docentes de Educación Secundaria, y de forma semejante entre los docentes de Educación Infantil y los docentes de Bachillerato con respecto a la dimensión de la evaluación integradora. En ambos casos, los docentes de la etapa Infantil presentaron puntuaciones superiores. Asimismo, también se hallaron diferencias entre los docentes de Educación Primaria y 
los docentes de Bachillerato, nuevamente con resultados superiores para los docentes que trabajan con alumnos de menor edad.

Respecto a la dimensión de evaluación contextualizada se obtuvieron diferencias estadísticamente significativas entre los docentes de Educación Infantil y los docentes de Secundaria y Bachillerato, con puntuaciones superiores para los docentes de Educación Infantil, confirmando así, de nuevo, la hipótesis de partida.

También se cumple la hipótesis respecto a la dimensión formativa de la evaluación, pues los docentes de cursos superiores, esto es, Secundaria y Bachillerato, presentaron puntuaciones más elevadas respecto a las etapas de Infantil y Primaria.

Finalmente, y para la dimensión de la evaluación personalizada se hallaron diferencias significativas entre los docentes de la etapa de Educación Infantil y Primaria con respecto a la etapa de Bachillerato.

En las dimensiones de evaluación reflexiva y evaluación democrática no se encontraron diferencias significativas. Los datos se recogen en la Tabla 6.

Tabla 6. Perfiles en función de la etapa educativa

\begin{tabular}{|c|c|}
\hline DIMENSIONES & $\begin{array}{c}\text { DIFERENCIAS ESTADÍTICAMENTE } \\
\text { SIGNIFICATIVAS }\end{array}$ \\
\hline Evaluación integradora & $\begin{array}{c}\text { E. I. con ESO y Bachillerato y E.P. con } \\
\text { Bachillerato. } \\
(\mathrm{F}=9.969, \mathrm{p}=0.000)\end{array}$ \\
\hline Evaluación contextualizada & $\begin{array}{c}\text { E. I. con ESO y Bachillerato. } \\
(\mathrm{F}=11.527, \mathrm{p}=0.000)\end{array}$ \\
\hline Evaluación formativa & $\begin{array}{c}\text { E.I. y E.P. con ESO y Bachillerato. } \\
(\mathrm{F}=22.339, \mathrm{p}=0.000)\end{array}$ \\
\hline Evaluación reflexiva & No se hallaron diferencias significativas \\
\hline Evaluación democrática & No se hallaron diferencias significativas \\
\hline Evaluación personalizada & $\begin{array}{c}\text { E.I. y E.P. con Bachillerato. } \\
(\mathrm{F}=5.914, \mathrm{p}=0.000) .\end{array}$ \\
\hline
\end{tabular}

\section{DISCUSIÓN}

El término evaluación genera en los docentes y profesionales de la educación diversas manifestaciones y se halla asociado a distintos procedimientos. En este sentido, se planteó el estudio de dichas concepciones en función del sexo, edad, titulación y años de experiencia docente, analizadas a través del Cuestionario de Evaluación Integral, estructurado en 6 dimensiones: integradora, contextualizada, formativa, reflexiva, democrática y personalizada.

Aunque la hipótesis de partida no consideraba diferencias estadísticamente significativas en función del sexo de los docentes, los resultados parecen apuntar una visión más tradicional en los docentes varones, asociada a la importancia de determinados momentos en los que debe realizarse la evaluación, frente a una visión más integradora, contextualizada y personalizada en las mujeres quienes consideran la necesidad de participación de diversos aspectos evaluativos. Respecto al propósito y los procedimientos de evaluación, 
no se hallaron diferencias entre sexos. Aunque se trata de una reducida muestra de estudio, las diferencias halladas deberían ser analizadas con mayor detalle con objeto de especificar posibles prácticas diferenciadas en función del sexo del profesorado. En este sentido, un estudio reciente llevado a cabo por Blanco y González (2010) halló diferencias entre maestros y maestras de Educación Primaria en sus prácticas de evaluación, siendo las maestras quienes, en mayor medida que los maestros, realizaban correcciones y supervisiones a lo largo del proceso de aprendizaje de los alumnos.

Respecto a la formación y etapa docente a la que pertenecían los participantes, los docentes con formación superior asociaron el término evaluación integral preferentemente a la idea de evaluación formativa y, por tanto, a la necesidad de evaluar en distintos momentos del proceso educativo. Además, consideraron en menor grado la evaluación de diversos aspectos del alumno y, en este sentido, es probable que se centren en características aptitudinales más que en elementos de personalidad o relacionados con sus intereses. Además, también valoraron en menor medida elementos contextuales, tales como el currículo, el centro o la familia, en sus actividades de evaluación, a diferencia de docentes de etapas inferiores. Se trata, por tanto, de una concepción más tradicional de la evaluación.

Frente a ellos, los docentes con formación media consideraron la evaluación vinculada a otros elementos y características del alumno, teniendo en cuenta una mayor variedad de técnicas y procedimientos que sus compañeros de etapas superiores. Estos resultados permiten concluir que la formación recibida por los maestros, es decir, los docentes diplomados, corresponde con una concepción más amplia de la evaluación que la recibida por los licenciados y especialistas en sus respectivas materias. Sin embargo, es preciso recordar que los docentes de etapas superiores indican sentir ciertas presiones, tanto sociales como educativas, vinculadas al logro de determinados éxitos académicos que pueden favorecer prácticas más tradicionales. Así, y aunque no se hallaron diferencias en las concepciones sobre evaluación entre los docentes de Educación Secundaria Obligatoria y Bachillerato, otros estudios hallaron tales diferencias. Concretamente, Pérez y Carretero (2009) observaron que los docentes de Secundaria centraban su interés en las actitudes y procedimientos de los alumnos, mientras que los docentes de Bachillerato centraron sus evaluaciones en los contenidos, primando los procesos de calificación, sin duda determinados por la necesidad de preparar a los alumnos para las Pruebas de Acceso a la Universidad.

Por otro lado, los resultados obtenidos en función de la edad de los docentes participantes ponen de manifiesto las distintas concepciones de evaluación asociadas a esta variable. Son los docentes más jóvenes quienes presentan visiones de la evaluación más flexibles (diversos aspectos a evaluar, evaluación de distintas áreas y ámbitos en los alumnos, estudio de elementos vinculados con las familias y el contexto, análisis del centro y del aula, etc.), claramente distantes con los docentes de mayor edad para quienes la idea de evaluación integral entronca con consideraciones asociadas a un único contexto de evaluación y con un número más limitado de técnicas a emplear y elementos a valorar. Cabe señalar que estos datos son nuevamente consecuentes con los hallados por Blanco y González (2010) en el estudio ya mencionado, quienes identificaron dos perfiles de docentes en función de su práctica evaluadora. Así, los docentes con menos años de experiencia ofrecieron un concepto de evaluación a lo largo de todo el proceso de aprendizaje y vinculado esencialmente a procedimientos y actitudes, frente a los docentes 
más experimentados quienes concedían mayor prioridad a los contenidos conceptuales. Resultados similares son concluyentes también con los datos obtenidos por Martín et al. (2006) y Bautista, Pérez y Pozo (2011). Sin duda, estos resultados son una interesante fuente de análisis e investigación pues cabe preguntarse sobre esta transformación que se produce en la conceptualización de la evaluación a lo largo de los años. A este respecto, Bautista, Pérez y Pozo (2011) reflexionan considerando que estas diferencias pueden explicarse, por un lado, por un posible cambio generacional, de modo que ambos grupos docentes pudieran pertenecer a distintas "culturas de aprendizaje" y, por tanto, mantuvieran distintas concepciones de la evaluación. La práctica y rutina diaria, obviamente acumulada en los docentes de mayor edad, también podría generar un efecto en la consideración de la evaluación, de tal forma que los años de experiencia permitan a los profesionales "simplificar" sus concepciones a medida que aumenta su práctica docente.

Así pues, las consideraciones de los docentes sobre la evaluación, recogidas a través de la aplicación del Cuestionario de Evaluación Integral (CEI), varían en función de su experiencia profesional, edad y sexo. Dado que el CEI nace de las teorías implícitas que los docentes tienen sobre la evaluación, es preciso analizar en qué medida dichas concepciones se ajustan a las prácticas reales llevadas a cabo. Del mismo modo que las teorías implícitas sobre la inteligencia responden a un docente más o menos flexible, preocupado por los resultados o por los procesos, que interpreta el esfuerzo como falta de capacidad o como condición para mejorar las capacidades y que, por tanto, busca interacciones que evitan la información negativa o, por el contario, ofrezcan información para aumentar el conocimiento (Dweck, 1995), las concepciones sobre la evaluación también pueden responder a un docente más o menos sensible hacia los elementos que configuran y participan en su toma de decisiones sobre la evaluación.

Sería, por tanto, deseable profundizar en el conocimiento de dichas concepciones con objeto de comprender la manera en que dichas prácticas se llevan a cabo y analizar las consecuencias de las mismas permitiendo proponer ámbitos de evaluación más amplios que favorezcan una evaluación multidimensional del alumno.

Aunque mejorar las prácticas educativas es un reto de gran dimensión, es importante iniciar procesos reflexivos con la participación de los docentes, así como estimular y reforzar todo intento de mejora desde la comunidad educativa, pues los grandes cambios siempre se inician con pequeños movimientos, y ningún proceso de cambio, y con más motivo en el entorno educativo, puede llevarse a cabo sin la participación y consenso de sus componentes. No podemos olvidar, por tanto, que los docentes son, sino el principal, uno de los factores clave en la educación (Enkvist, 2010).

Así, incluso los mejores profesores (aquellos que obtuvieron un mejor evaluación del desempeño según el estudio de Vergara, 2011) consideran que no siempre se corresponde la teoría con la práctica, y que las actividades y rutinas del día a día, probablemente relacionadas con las exigencias administrativas y la falta de tiempo, fuerzan prácticas mecánicas de evaluación a través de la aplicación de instrumentos, dejando de lado la evaluación de procedimiento y centrándose principalmente en los conocimientos. Por tanto, es preciso también repensar las tareas, las exigencias y responsabilidades que los docentes deben asumir. 


\section{REFERENCIAS BIBLIOGRÁFICAS}

Bautista, A., Pérez, M.P y Pozo, I. (2011). Concepciones de profesores de piano sobre la evaluación. Revista de educación, $n$, 355, 443-466.

Blanco, M.E. y González, I. (2010). Prácticas evaluativos del profesorado de Primaria, condicionantes de su actividad docente. Bordón, vol. 62, n. 1, 29-47.

Brown, G. T. L. (2004). Teachers' conceptions of assessment: implications for policy and professional development. Assessment in Education, vol. 11, n. 3, 301-318.

Cabezas, D. y Carpintero, D. (2006). Teorías Implícitas sobre la inteligencia en docentes. EduPsykhé, vol. 5, n. $1,129-142$.

Cabezas, D., González, C. y Carpintero, E. (2009). Evaluar la evaluación. Cuestionario sobre prácticas de evaluación. EduPsykhé, vol. 8, n. 1, 51-61.

Carpintero, E. y Cabezas, D. (2007). Análisis comparativo de las Teorías Implícitas sobre la Inteligencia elaboradas por docentes y estudiantes del ámbito educativo. EduPsykhé, vol. 6, n. $1,109-121$.

Coll, C., Martín, E. y Onrubia, J. (2001). La evaluación del aprendizaje escolar: dimensiones psicológicas, pedagógicas y sociales. En C. Coll, J. Palacios y A. Marchesi (Coords.), Desarrollo psicológico y educación, II (pp. 549-572). Madrid: Alianza.

De la Orden, A. (1989). Investigación cuantitativa y medida en educación. Bordón: Revista de Orientación Pedagógica, n. 41, 217-236.

Dweck, C.S. (1995). Implicit theories as organizers of goals and behavior. En P.M. Gollwitzer y J.A. Bargh (Eds.), The psychology of action: Linking cognition and motivation to behavior. Nueva York: Guildford Press.

Enkvist, I. (2010). El éxito educativo finlandés. Bordón, vol. 62, n. 3, 49-67.

Fleitman, J. (2007). Evaluación integral para implantar modelos de calidad. Mexico: Editorial Pax.

García, A., Barría, A., Prieto, B., Herrera, G. y Vázquez, D. (2006). Proyectos de Inversión: evaluación integral. Un enfoque práctico. Edición electrónica. Texto completo en www.eumed. net/libros/2006c/218/

Ley Orgánica 2/2006, de 3 de mayo, de Educación. BOE 106, 4 de mayo de 2006.

Litwin, E. (2005). La evaluación: campo de controversias y paradojas o un nuevo lugar para la buena enseñanza. En A. Camilloni et al. (2005). La evaluación de los aprendizajes en el debate didáctico contemporáneo (pp. 11-34), Buenos Aires: Editorial Paidós Educador.

Martín, E., Mateos M., Martínez, P., Cervi, J., Pecharromán, A., y Villalón, R. (2006). Las concepciones de los profesores de primaria sobre la enseñanza y el aprendizaje. En J. I. Pozo, N. Scheuer, M. P. Pérez Echeverría, M. Mateos, E. Martín y M. de la Cruz (Comps.), Nuevas formas de pensar la enseñanza y el aprendizaje: las concepciones de profesores y alumnos (pp. 143-159). Barcelona: Graó.

Pérez Juste, R. (2002). La evaluación de programas en el marco de la educación de calidad. XXI, Revista de Educación, n. 4, 43-76.

Pérez, L y González, D.A. (2011). "Dime Cómo Evalúas y te Diré Qué Enseñas". Un Análisis Teórico sobre las Relaciones entre la Evaluación del Aprendizaje y la Enseñanza-Aprendizaje de la Justicia Social. Revista Iberoamericana de Evaluación Educativa, vol. 1, n. 4, 135-148.

Pérez, M.L. y Carretero, M.R. (2009). La evaluación del aprendizaje en Educación Secundaria: análisis de un proceso de cambio. Límite. Revista de Filosofía y Psicología, vol. 19, n. 4, 93-126.

Perrenoud, P. (2008). La evaluación de los alumnos. De la producción de la excelencia a la regulación de los aprendizajes. Entre dos lógicas. Buenos Aires: Colihue.

Prieto, M. y Contreras, G. (2008). Las concepciones que orientan las prácticas evaluativas de los profesores: un problema a develar. Estudios Pedagógicos, vol. 34, n. 2, 245-262.

Rosales, C. (2000). Evaluar es reflexionar sobre la enseñanza. Madrid: Narcea. 
Salinas, D. (2002). ;Mañana examen! La evaluación: entre la teoría y la realidad. Barcelona: Graó. Sanmartí, N. (2007). Diez ideas clave. Evaluar para aprender. Barcelona: Grao.

Santos, M.A. (2003). Una flecha en la diana. La evaluación como aprendizaje. Madrid: Narcea. Vergara, C. (2011). Concepciones de evaluación del aprendizaje de docentes destacados de Educación Básica. Actualidades Investigativas en Educación, vol. 1, n. 11, 1-30. Recuperado en http://revista.inie.ucr.ac.cr/uploads/tx_magazine/concepciones-evaluacion-aprendizaje-docentes-vergara.pdf 